This document is published in:

Andre Ponce de Leon F. de Carvalho et al. (eds.), 2010. Distributed Computing and Artificial Intelligence: 7th International Symposium

(Advances in Intelligent and Soft Computing, 79), Springer, pp. 309-316.

DOI: 10.1007/978-3-642-14883-5_40

(C) 2010 Springer-Verlag Berlin Heidelberg 


\title{
Multi-Sensor and Multi Agents architecture for indoor location
}

Gonzalo Blázquez Gil, Antonio Berlanga de Jesús, and José M. Molina

Lopéz

\begin{abstract}
This paper aims to present a new architecture to provide location services using multiple communication technologies such as Wifi, UWB, RFID and so on. Firstly, it will explain the advantages of multi sensor architecture against to use unique indoor location system and the reasons which led us to take this solution. Besides, this paper discusses the suitability of using ontologies for modeling message structure to locate in context-aware services platforms. This message will be described based on the concept of Asterix format used in aerospace multi-sensor communications.
\end{abstract}

\section{Introduction}

Context-aware Systems allow to develop a new kind of location-aware mobile applications in different sectors such as healthcare, military, emergencies, and recently retail and agriculture $[2,5]$. These applications could be represented as a context based scenario where there are individuals who require a satisfaction of their needs and there are providers who can solve these lacks. In context-aware computing, context is any information that can be used to characterize the situation of an entity (i.e. a person, computing device, or other). In this case, the architecture proposed focused on improving the location performance.

Positioning (also called Location aware) has been a main factor in the development of context applications. Location Awareness in general describes applications, which change their behavior according to the position of the user [3]. Location-aware enables new kinds of services and applications [4].

Applied Artificial Intelligence Group, Universidad Carlos III de Madrid, Avd. de la Universidad Carlos III, 22, 28270, Colmenarejo, Madrid, Spain \{gonzalo.blazquez, antonio.berlanga, josemanuel.molina\}@uc3m.es http://www.giaa.inf.uc3m.es 
In outdoor environments GPS provides an effective solution to determine the location of mobile devices. However, in GPS-denied areas such as urban, indoor, and subterranean environments, unfortunately, an effective location technique does not exist. In this paper, it is presented an architecture which use multiple wireless technologies (UWB, Wifi, RFID, Bluetooth) [7] to provide location services. Hence, it is difficult to develop a location-aware application without making assumptions about the indoor location technology. So, it is possible to combine multiple indoor location systems to meet applications requirements.

Multi-sensor integration is becoming an essential aspect of indoor location systems [8]. Merge data from multiple indoor location systems and gather that information in order to achieve inferences, which will be more efficient and more accurate than if data were obtained of a single source. Applying Multi-sensor concepts could be improved the system performance, whether it is in coverage, availability or accuracy [1].

In [9] is described a new Ambient Intelligent Platform based on Multi Agent Systems (MAS) where location is included in the Locator Agent. Based on this architecture, it will be proposed that the Locator as Fusion architecture (Fusion Agent) and each Indoor Location System (ILS) is an agent which sends information about the location of each user to Fusion agent. To make easy the fusion process, fusion agent needs to know the message structure of each ILS, since this structure is different in each ones, it is necessary to standardize the structure message to ILS message structure.

Researchers in agent communication languages cite three important elements in MAS interaction [6]: (i) a common agent communication language and protocol; (ii) a common format for the content of communication and (iii) a shared ontology. The first two points are solved with declarative agent languages such as KQML or FIPA. Nevertheless, we will focus on creating an ontology which represents the conceptualization of positioning based on Asterix (All Purpose Structured Eurocontrol Surveillance Information Exchange) format, used in air navigation. In this paper, it is presented GONZ, Global Ontology for Indoor Localization, which aims to standardize the communication between ILS agent and Fusion Agent for indoor environments.

In this paper we propose an architecture which processed the measurement data received from indoor location system and besides, enhances the association process using the attributes of GONZ format. This paper is organized as follows: Section 2 describes the new fusion architecture for indoor applications. Section 3 presents a new ontology for indoor localization based on Asterix. Section 4 provides some concluding comments and future researches lines. 


\section{Multi-agent Fusion Architecture}

In this section, we will describe the Fusion architecture based on Multi Agent system. Figure 1 represents a context-aware Multi Agent system architecture for heterogeneous domain used by Venturini et al [9] to describe Ambient Intelligent Platform based on Multi Agent Systems. Locator Agent, composed of a single indoor location system, is responsible to locate each user agent.

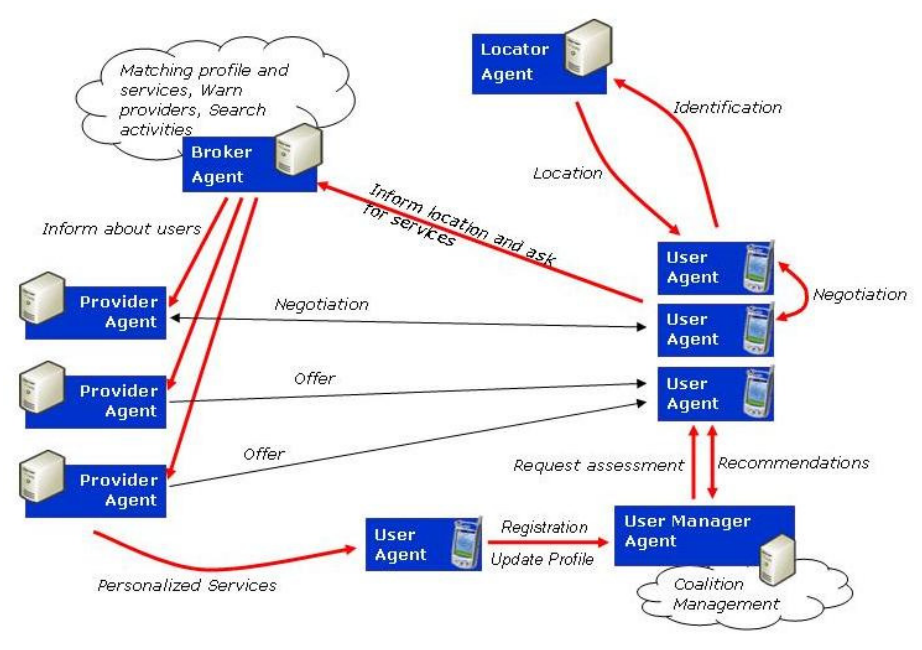

Fig. 1 Context-aware Multi Agent system architecture for heterogeneous domain.

The structure of this architecture is composed of service provider agents (including Locator agent) and User agents. Provider agent offers services (Location, advertisement, weather information and whatever you want) to User Agent. This architecture presents several disadvantages in indoor environments due to indoor location issues: Multipath, reflexions, refraction, etc.

Multi-sensor architecture improves the precision, correcting systematic errors of some technologies with those ones they have not. Besides, if the Context aware architecture is able to obtain user position for more than one technology (Locator Agent) the system availability is improved. Finally, user availability is improved if multiple technologies could offer services to them. So, users need not to carry on a different mobile device in each environment. For all these reasons, it is interesting to design a new concept of Locator agent based on Multi-sensor fusion architectures.

Normally, indoor location systems run in their own server, therefore, a distributed architecture is needed. Multi agent system offers good features (Modularity, scalability and robustness) to solve distributed problems against other architectures like server-client or ad-hoc systems which present im- 
portant lacks. The modularity of MAS permits make easy to develop new functions. For example, if new Indoor location Systems is added to the architecture, it is only necessary to create an agent which carry out the message format proposed in the last section.

The architecture proposed for the Locator agent is based on a layered architecture for fusion process as illustrated in figure 2, with separate layers for raw sensor data (ILS API), for features extracted from ILS (Locator Layer), and for context derived from ILS (Fusion Layer). Each component is developed as an agent the first one is the (i) Fusion Center, which integrates data from all technologies and provides a location context for each user. The second one, called (ii) Locator layer, is responsible for providing location measurements to Fusion Center (Locator Agent in Figure 1).

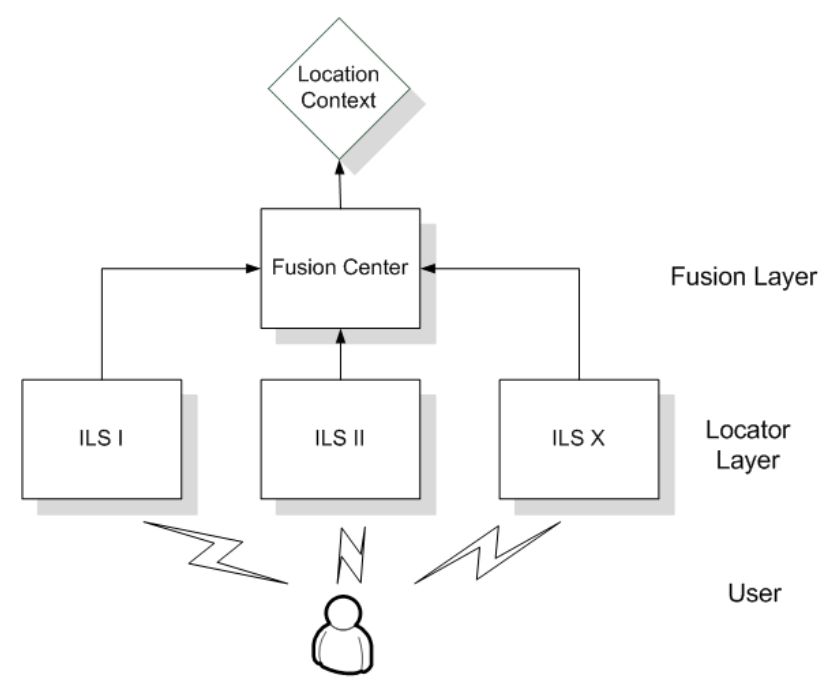

Fig. 2 Locator agent architecture

\subsection{Fusion layer}

The core function is collect and fuse information from available indoor location system (ILS agent) to provide user location context of each user. The fusion center received periodically (each $\mathrm{T}$ seconds) the detected data from ILS. Fusion layer could be split in other three layers (also see Figure 3). 
Multi-Sensor and Multi Agents architecture for indoor location

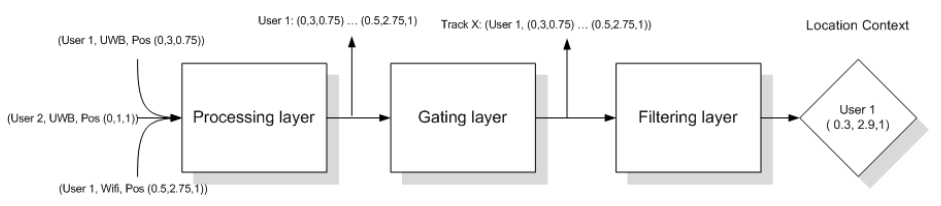

Fig. 3 Architecture of Fusion layer.

\subsubsection{Processing layer}

Processing layer is an agent which aims to receive measurement information of each ILS agent and starts the fusion process. Firstly, the new user position is stored in a vector structure which contains the last valid positions of this target. Then, the new measurement is transformed to unify coordinates values with respect to the same global position (it is defined in the next section Global Ontology for Indoor location). Each measurement is preprocessed depending on the position of the ILS which generates it. To conclude, the structure will pass to the next layer and the fusion process continues.

\subsubsection{Gating layer}

Gating layer aims to evaluate the chance of a new measurement will become a track and besides, search for associations between measurements and tracks, managing the structure of new measurements and the non-assigned plots of previous periods.

\subsubsection{Filtering layer}

The filtering layer correlates the tracks belonging to the same located-object (user device) from various sources (ILS) and it aims to manage of tracks: Create, delete or fuse tracks with similar features.

\subsection{Locator layer}

Since indoor location systems tend to be proprietary and only provide us a communication protocol or API in $\mathrm{C} \sharp$, java, $\mathrm{C}$, etc to access to measurement, it is necessary to create a wrapper which aims to obtain, process and send to fusion layer measurements in a common language. Therefore, Locator layer is an agent which allows Fusion center works together that normally could not because of each ILS provide a different message structure. Hence, the Locator layer is responsible for transforming data into appropriate form. For instance, 
if a concrete ILS send position values in inches but other one send position in meters, the Locator layer would be responsible for unit conversion.

\subsection{Multi Agent System Interaction}

Interaction diagram (AUML Interaction Protocol Diagrams) describes better the Multi Agent Architecture proposed, with the advantage to identify clearly several agents roles. The figure below shows three agents, two of them belonging to the Location layer (ILS I and ILS II) and the other one belonging to Fusion Layer (Processing Layer).

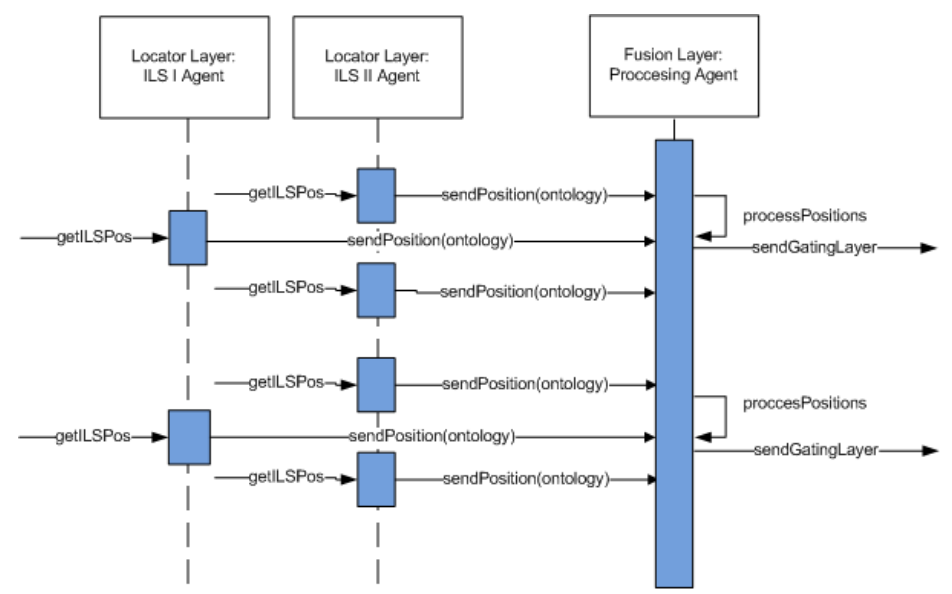

Fig. 4 Interaction Diagram of Location.

ILS agents receive location information from each ILS, process this information and send location information to the Fusion layer, These actions are performed periodically, according to the sampling frequency each Indoor Location System ( In this case, ILS II sends measurement faster than ILS I). Processing Agent also works periodically but the frequency does no have to be the same as the ILS Agent. In this case, Processing agent receive user position measurement, store it and send it to the next layer of the of the Fusion Center (Gating layer)

\section{Global Ontology for Indoor Location}

To allow interoperability between Fusion Layer and Locator layer, it is necessary that the location context terminology is understood by all participat- 
ing devices. In this section we propose an adaptable and extensible location context ontology (Global ontology for indoor location, GONZ) for which describes the knowledge in the communication process between agents. GONZ format presents the same structure message for all kind of indoor location system since the differences between every sensor are not considerable.

Other important point is that this message structure contains high level parameters since several ILS does not provide raw measurement (TDoA, azimuth, RSSI, etc). In this work we only have considered parameters in the format message for systems that provide the height and those ones that do not. Later, if new technologies were developed and provides remarkable features to fuse, it could create a new message format to GONZ standard.

\begin{tabular}{|l|}
\hline Height known \\
\hline Identification \\
\hline Time measurement \\
\hline Sampling frequency \\
\hline [Origin of coordinates] \\
\hline Pos X \\
\hline Pos Y \\
\hline Pos Z \\
\hline Error \\
\hline
\end{tabular}

\begin{tabular}{|l|}
\hline Height unknown \\
\hline Identification \\
\hline Time measurement \\
\hline Sampling frequency \\
\hline [Origin of coordinates] \\
\hline Pos X \\
\hline Pos Y \\
\hline Error \\
\hline
\end{tabular}

Fig. 5 GONZ format message for height known and unknown.

Then, we will specify the principal parameters of the Global Ontology for Indoor Location format:

- Identification: Value which identified a track unambiguously, this identifier is composed for two strings: $i d_{\text {track }}-i d_{\text {devicelocator }}$.

- Time measurement (UTC): The time in UTC format of the last measurement taken.

- Sampling frequency (ms): Defines the time between two consecutive measurements.

- Origin of coordinates: In this attribute is stored (If it is possible) in Geographic coordinates or a common representation the origin of coordinate's of the indoor location system.

- Position X, Y, Z coordinates (m): Vector which contains the $\mathrm{x}, \mathrm{y}$, z position in meters of the user.

- Error: Estimated error of the measurement given.

An ontology represents a hierarchy structure of the environment knowledge where now the root node is GONZ and its child nodes are a concrete message (Height know message and Height unknown message). If new kind of message was created, a new leaf node could be added in the actual structure of the ontology. E.g. A new message structure could be created for a GPS devices or GSM devices since none of them are valid in the current standard. Then, it is necessary to create a new child node whose parents (GONZ, Height ${ }_{\text {known }}$ or Height $t_{\text {unknown }}$ ) depending on the features that new technology has. 


\section{Conclusions}

This paper presents a Multi agent architecture to enhance the location in indoor environments with different indoor location technologies. The proposed architecture is responsible to obtain, process and fuse the measurements of each indoor location system and provide the result of fusion as location context.Besides, a structure message (GONZ) is defined for describing the communication between each ILS with the Fusion Center.

The principal advantages which offers this architecture is a highly interchangeable and adaptable system. If a new indoor technology will be developed, it will not involve major changes in its architecture. Only it will affect the inclusion of a new kind of message in the Global Ontology for Indoor Location.

\section{Acknowledgments}

This work was supported in part by Projects CICYT TIN2008-06742-C0202/TSI, CICYT TEC2008-06732-C02-02/TEC, CAM CONTEXTS (S2009/TIC1485) and DPS2008-07029-C02-02

\section{References}

1. S. Aparicio, J. Pérez, P. Tarrío, A. Bernardos, and J. Casar. An indoor location method based on a fusion map using Bluetooth and WLAN technologies. In International Symposium on Distributed Computing and Artificial Intelligence 2008 (DCAI 2008), pages 702-710. Springer.

2. C. Becker and F. Dúrr. On location models for ubiquitous computing. Personal and Ubiquitous Computing, 9(1):20-31, 2005.

3. G. Chen and D. Kotz. A survey of context-aware mobile computing research. Technical report, Citeseer, 2000.

4. K. Cheverst, N. Davies, K. Mitchell, and A. Friday. Experiences of developing and deploying a context-aware tourist guide: the GUIDE project. In Proceedings of the 6th annual international conference on Mobile computing and networking, pages 20-31. ACM, 2000.

5. S. Clarke. Context-aware trails. 2004.

6. T. Gruber et al. A translation approach to portable ontology specifications. Knowledge acquisition, 5:199-199, 1993.

7. P. Hii and A. Zaslavsky. Improving location accuracy by combining WLAN positioning and sensor technology. Monash University, AU.

8. U. Leonhardt and J. Magee. Multi-sensor location tracking. In Proceedings of the 4 th annual ACM/IEEE international conference on Mobile computing and networking, page 214. ACM, 1998.

9. J. C. Verónica Venturini and J. M. Molina. An Ambient Intelligent Platform based on Multi-Agent System. pages 1-9, 2008. 